\title{
Providing updated guidelines for the management of rheumatic diseases in Italy with the ADAPTE methodology: a project by the Italian Society for Rheumatology
}

\author{
M. Manara ${ }^{1,2}$, N. Ughi ${ }^{1,2}$, A. Ariani ${ }^{1,3}, A$. Bortoluzzi $^{1,4}$, S. Parisi ${ }^{1,5}$, I. Prevete ${ }^{1,6}$, \\ G. Carrara' ${ }^{1}$, A. Zanetti', F. Rumi' ${ }^{1}$, C.A. Scirè̀ ${ }^{1,4}$ \\ ${ }^{1}$ Study Centre, Italian Society for Rheumatology, Milan, Italy; ${ }^{2}$ Rheumatology Department, \\ Centro Specialistico Ortopedico-Traumatologico Gaetano Pini - CTO, Milan, Italy; ${ }^{3}$ Department of Medicine, \\ Internal Medicine and Rheumatology Unit, University Hospital of Parma, Italy; ${ }^{4}$ Department of Medical \\ Sciences, Rheumatology Section, University of Ferrara, Azienda Ospedaliero-Universitaria Sant'Anna \\ di Cona, Ferrara, Italy; ${ }^{5}$ Rheumatology Unit, Azienda Ospedaliera Città della Salute e della Scienza di Torino, \\ Italy; ${ }^{6}$ Rheumatology Unit, Azienda Ospedaliera Santo Spirito, Rome, Italy
}

\section{INTRODUCTION}

D eveloping ex novo clinical practice guidelines (CPGs) and recommendations is time-consuming and requires a significant effort in terms of human and economic resources. Moreover, CPGs on the same topic may be produced by different scientific societies, with a duplication of efforts. On the other side, the need for up-to-date guidelines in clinical practice implies the continuous updating of existing recommendations based on evidence emerging from new studies (1).

The ADAPTE collaboration, an international collaboration of guideline developers, researchers and clinicians who aim to promote the development and use of clinical practice guidelines through the adaptation of existing guidelines, has provided a well-defined methodology which enables production of updated guidelines based on existing ones, saving resources and time $(2,3)$. The ADAPTE process is defined as a systematic approach to adapting guidelines produced in one setting for use in a different cultural and organizational context. The process which leads to adapted guidelines implies not only the extrapolation and synthesis of evidence emerging from previous guidelines, but also the adaptation to a different setting, preserving the evidence on which original recommen- dations are based when accommodating for local needs. The detailed reporting of each step guarantees the transparency and reproducibility of the process and the validity of the adapted guidelines. The search for an external validation may foster the applicability and acceptability in the local context. In April 2017, the Study Centre of the Italian Society for Rheumatology (SIR) resolved to use the ADAPTE methodology with the aim of providing updated guidelines on the management of diseases of concern to the SIR members. The Study Centre planned to develop adapted guidelines regarding selected diseases and to perform a periodic update of each thematic area. The project was approved by the Steering Committee of the SIR.

\section{THE ADAPTE METHODOLOGY IN THE DEVELOPMENT OF THE SIR GUIDELINES}

The process went through three phases: a set-up phase, an adaptation phase and a final phase (Figure 1).

\section{Set up phase}

At the beginning, a scoping review was performed to assess whether the adaptation was feasible for the topics identified by the Steering Committee of the SIR. Thus, a non-systematic search of literature was
Corresponding author: Maria Manara Reumatology Department, Centro Specialistico Ortopedico-Traumatologico Gaetano Pini CTO

Piazza Cardinal Ferrari, 1 20122, Milan, Italy

E-mail:maria.manara@gmail.com 


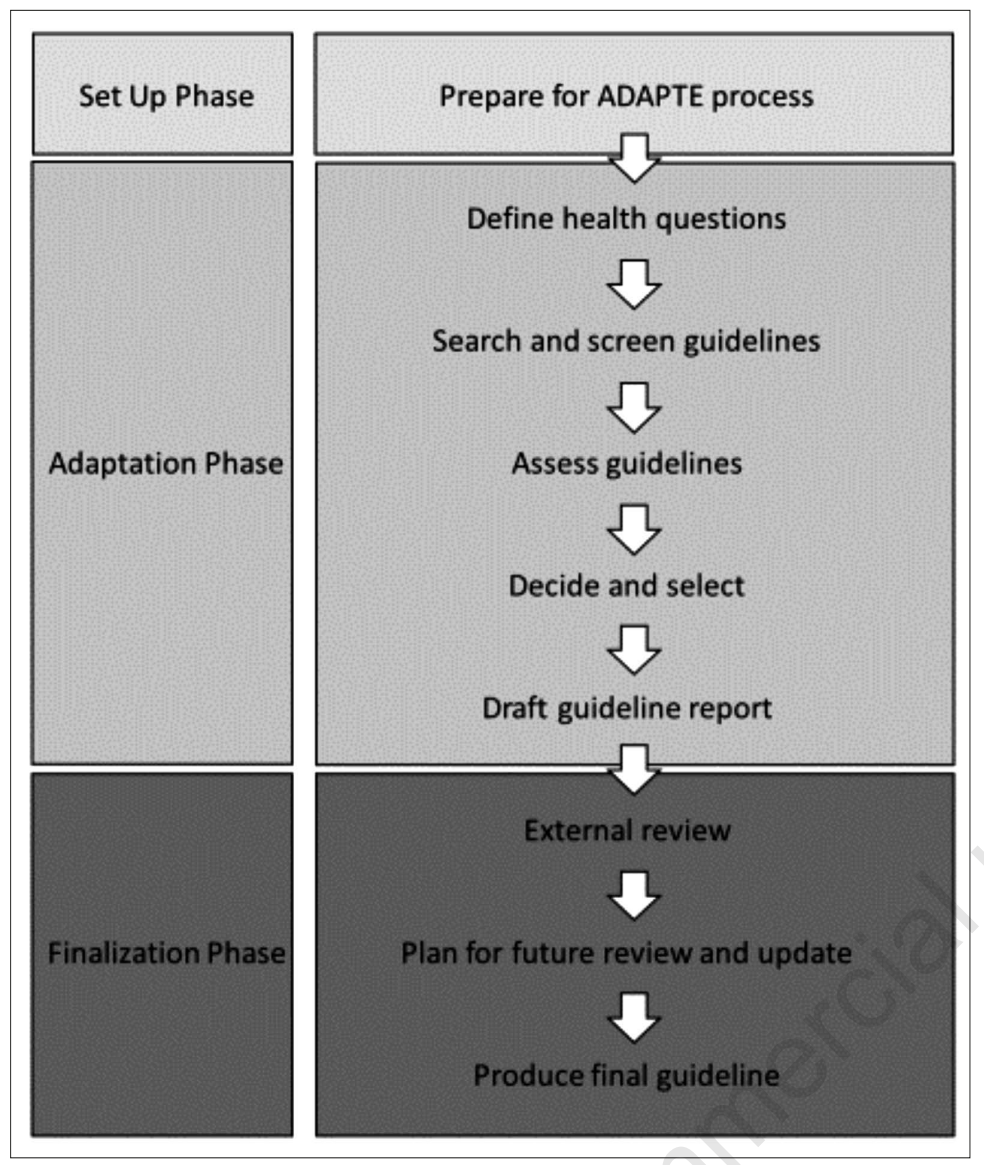

Figure 1 - Summary of the ADAPTE process. (Reproduced and adapted with permission from: The ADAPTE collaboration (2009). The ADAPTE process: resource toolkit for guideline adaptation. Version 2.0. Available from: http://www.g-i-n.net).

conducted to assess the existence of recent international guidelines to support adaptation (4).

For each of the clinical issues proposed by the Steering Committee of the SIR, a working group was set up and two members of the Study Centre were responsible of the development of the CPG under the supervision of the project coordinator. The working group drafted the protocol of the adaptation plan and established the schedule for the process.

For each clinical issue, a panel of experts was identified for the task of the adaptation process. The panel was composed of members of the SIR Study Centre, on account of their methodological knowledge, and of three clinicians who were identified by the Steering Committee of the SIR for their recognized expertise in the field. Each member of the panel was required to sign a conflict of interests declaration.

\section{Adaptation phase}

After the target of each adapted CPG was specified, a list of key clinical questions covering all areas of interest of the CPG was proposed by the working group and sent to each member of the panel for evaluation and approval.

A systematic search of the literature was performed to identify all the CPGs concerning the clinical issue that was the object of the adaptation and published within a pre-defined timeframe. The search was performed on at least two databases (including Medline and Embase) and integrated with a search of grey literature via online guidelines databases (e.g. the Agency of Healthcare Research and Quality database, https://www.guideline.gov/) and internet gatekeepers (e.g. Google). The search strategy was defined on the base of clinical issue and the key words were related to the disease of interest in combination with the terms identifying guidelines, recommendations, and consensus.

The inclusion criteria for retrieved guidelines were pre-defined in the protocol, concerning population, year of publication, language and guideline developer. The panel agreed to include only evidence-based guidelines produced by relevant national or international scientific societies involved in the management of the disease that was the object of the guideline (primarily the American College of Rheumatology and the European League Against Rheumatism) or by the SIR. The identification and selection of CPGs responding to inclusion criteria was performed by two independent reviewers and in case of disagreement a third member of the panel was involved to solve it by discussion; all the phases of the process were recorded and reported in a flow-chart.

For each CPG, two members of the panel independently performed a quality assessment with the last version of the Appraisal of Guidelines Research and Evaluation instrument (AGREE II) (5). The AGREE II 
allows a comprehensive evaluation of quality, in terms of both the methodology of the process of development of the CPG and its reporting, though not its clinical content. The instrument is made up of 23 items organized within 6 domains; each item of the instrument is rated on a 7-point scale and a global score for each domain can be calculated. At the end, the evaluator is asked to give an overall rating of the quality of the CPG and a global indication as to whether he/she would recommend the guideline in clinical practice.

Each evaluator was trained for use of the AGREE II instrument on the AGREE Web site before starting the quality assessment for the ADAPTE project (www.agreetrust. org). AGREE scores from both the evaluators were recorded for each CPG. The panel did not establish a threshold of AGREE score to allow the inclusion of the CPG in the adaptation process, but the AGREE score was considered to support the phase of extrapolation and adaptation of the final recommendations from the original CPGs. In the following phase, for each clinical question a matrix of recommendations was created, namely a table containing the recommendations reported in the original CPGs which were pertinent to the clinical question. In the matrix, the name of the source CPG, the year of publication, the AGREE score, and the level of evidence and the strength of the recommendation according to their original system for grading the clinical evidence were also reported for every recommendation extracted from the included CPGs. All the recommendations responding to the same clinical question were reported in the same matrix in order to allow direct comparisons between the CPGs and facilitate the discussion of the potential disagreements.

For the adaptation process, consistency among recommendations was evaluated in terms of consistency of search strategy, consistency of the body of evidence supporting every recommendation, and consistency between the interpretation of the evidence and the formulated sentences. Acceptability and applicability in the Italian context were judged by considering the availability of treatments and the organization of the national health system.

When the review and evaluation process was completed, the members of the panel gathered in face-to-face and web-meetings to draft a proposal for adapted recommendations. Original propositions were adopted, rejected or rephrased considering consistency, currency, quality and applicability of the source recommendations. In order to standardize different grading systems of evidence used across guidelines, the levels of evidence and the strength of the recommendations were reported according to the Oxford Levels of Evidence (6). The draft of the adapted CPG was approved by all the members of the panel.

Finally, the panel prepared a draft of the paper detailing the process of adaptation and reporting the adapted recommendations.

\section{Finalization phase}

In the finalization phase, each adapted CPG was externally reviewed by the target users of the recommendations. The panel of external reviewers was made up of rheumatologists with clinical expertise in the field of the CPG, other specialists involved in the multidisciplinary management of the clinical condition, a general practitioner, a healthcare professional, and authors of the original guidelines. The list of external reviewers was defined by the Steering Committee of the SIR.

The external reviewers were consulted by an online survey and were asked to rate each recommendation within the CPG in terms of scientific content and applicability, i.e. the use of the adapted recommendations in clinical practice. The ratings were recorded and incorporated in the final document.

A plan of dissemination was agreed upon with the SIR for the peer-review publication in the official journal of SIR, Reumatismo, and a plan for updating the guidelines was reported in the final document.

\section{CONCLUSIONS}

The ADAPTE methodology is a valid alternative to the de novo development of 
updated CPGs, since it optimizes the utilization of resources and preserves the quality, consistency and applicability of the adapted CPGs. The implementation of guideline adaptation by the SIR may provide updated guidelines for the needs of the clinical management of rheumatic diseases within the Italian health system in everyday practice.

\section{REFERENCES}

1. Shekelle PG, Ortiz E, Rhodes S, et al. Validity of the Agency for Healthcare Research and Quality clinical practice guidelines: how quickly do guidelines become outdated? JAMA. 2001; 286: 1461-7.
2. Fervers B, Burgers JS, Voellinger R, et al. The ADAPTE collaboration. Guideline adaptation: an approach to enhance efficiency in guideline development and improve utilization. BMJ Qual Saf. 2011; 20: 228-36.

3. The ADAPTE collaboration. The ADAPTE process: resource toolkit for guideline adaptation; 2009. Version 2.0. Available from: http:// www.g-i-n.net

4. Peters MD, Godfrey CM, Khalil H, et al. Guidance for conducting systematic scoping reviews. Int J Evid Based Healthc. 2015; 13: 141-6.

5. Brouwers M, Kho ME, Browman GP, et al. AGREE II: Advancing guideline development, reporting and evaluation in healthcare. Can Med Assoc J. 2010; 182: E839-842.

6. Oxford Centre for Evidence-Based Medicine. Levels of evidence; March 2009. Available from: http://www.cebm.net/?o=1116 